\title{
Topological superconductivity in carbon nanotubes with a small magnetic flux
}

\author{
Omri Lesser $\odot$, Gal Shavit $\odot$, and Yuval Oreg \\ Department of Condensed Matter Physics, Weizmann Institute of Science, Rehovot 76100, Israel
}

(Received 17 March 2020; revised manuscript received 4 May 2020; accepted 12 May 2020; published 1 June 2020)

\begin{abstract}
We show that a one-dimensional topological superconductor can be realized in carbon nanotubes, using a relatively small magnetic field. Our analysis relies on the intrinsic curvature-enhanced spin-orbit coupling of the nanotubes, as well as on the orbital effect of a magnetic flux threaded through the nanotube. Tuning experimental parameters, we show that a half-metallic state may be induced in the nanotube. Coupling the system to an Ising superconductor, with an appreciable spin-triplet component, can then drive the nanotube into a topological superconducting phase. The proposed scheme is investigated by means of real-space tight-binding simulations, accompanied by an effective continuum low-energy theory, which allows us to gain some insight on the roles of different terms in the Hamiltonian. We calculate the topological phase diagram and ascertain the existence of localized Majorana zero modes near the edges. Moreover, we find that in the absence of a magnetic field, a regime exists where sufficiently strong interactions drive the system into a time-reversal-invariant topological superconducting phase.
\end{abstract}

DOI: 10.1103/PhysRevResearch.2.023254

\section{INTRODUCTION}

Low-dimensional topological superconductors are unique states of matter, supporting Majorana fermions at the system's edges [1-4]. These zero-energy edge modes have non-Abelian exchange statistics, making them a very attractive platform for realizing quantum computation schemes [5,6]. Experimental evidence for the emergence of Majorana zero modes, at the ends of one-dimensional (1D) semiconducting nanowires with strong Rashba spin-orbit coupling (SOC) and induced Zeeman spin splitting, was observed in the form of zero-bias conductance peaks in several instances [7-11].

An alternative route to realizing 1D topological superconductivity is using carbon nanotubes (CNTs) [12] instead of semiconducting nanowires. CNTs are small-diameter tubes of rolled-up graphene, having exceptional electronic band structures and transport properties [13,14]. As opposed to nanowires, CNTs have a truly 1D nature, as their diameter $d$ is extremely small (of order $1 \mathrm{~nm}$ ). Moreover, being comprised entirely of carbon atoms, very clean CNTs may be fabricated, thus facilitating probing of their quantum properties [15].

These properties make CNTs an attractive platform for pursuing 1D Majorana fermions, and several schemes aimed at achieving those have been put forward [16-19]. The proposals mainly rely on the same ingredients available in the semiconducting-nanowires setups: a combination of proximity to an $s$-wave superconductor, SOC, and a Zeeman magnetic field. The latter, due to the low $g$ factor of the CNTs,

Published by the American Physical Society under the terms of the Creative Commons Attribution 4.0 International license. Further distribution of this work must maintain attribution to the author(s) and the published article's title, journal citation, and DOI. typically needs to be very large, which poses experimental challenges: high magnetic fields are not easily produced, and may also critically suppress superconductivity in the proximitizing substrate.

In this paper we present a scheme which allows us to circumvent the high Zeeman-energy problem, and realize topological Majorana zero modes without the need for any Zeeman splitting. Our scheme, depicted in Fig. 1, relies instead on an orbital effect caused by a magnetic flux threaded through the nanotube. When it is combined with the unusual SOC present in CNTs, and the breaking of the CNT's rotational symmetry (by, e.g., an external gate), the CNT can be tuned into a half-metallic state using relatively low magnetic fields. Then, proximitizing the CNT to a superconductor with a significant spin-triplet component in its Cooper-pairs wave function, a $p$-wave topological gap may open in the nanotube, hosting Majorana fermions near its edges. Thin films of transitionmetal dichalcogenides (TMDs) make excellent candidates for the superconducting substrate, having strong Ising SOC, favorable for pairing of electrons with their spin polarized in the TMD plane.

The presence of spin-triplet pairs in the superconducting substrate opens up another interesting possibility, as the interaction between electrons in the CNT heavily favors triplet pairing over the singlets. Then, in a regime with zero magnetic flux, strong enough interactions may allow one to tune the system into a time-reversal-invariant topological superconductor phase [20,21].

The rest of the paper is organized as follows. In Sec. II we introduce the theoretical model of our system, as well as the effective low-energy theory. Section III is dedicated to the spin-triplet proximity effect, and its implementation using superconducting TMDs. We show that a topological superconducting phase is supported by our model in Sec. IV. The presence of Majorana zero modes bound to the edges 


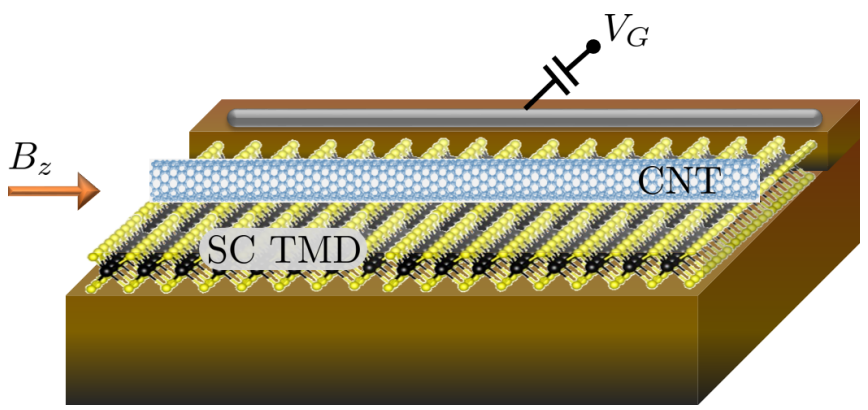

FIG. 1. Our proposed setup for realizing topological superconductivity in a CNT. We apply a magnetic field $B_{z}$ parallel to the CNT, and use a nearby metallic gate (gray) with voltage $V_{\mathrm{G}}$ to tune its chemical potential. In the presence of SOC and rotational-symmetry breaking (inherent in our setup), these allow us to tune the CNT to a half-metallic point. Thus, proximity coupling to a superconducting TMD substrate may open a topological gap in the CNT, hosting Majorana states at the edge.

is demonstrated in Sec. V. The conditions for realizing a topological phase without magnetic flux are presented and discussed in Sec. VI. Weconclude our findings in Sec. VII.

\section{MODEL FOR THE CNT}

We consider a tight-binding model of the $\pi$ electrons of a cylindrically rolled-up graphene lattice comprising the
CNT. The CNT may be classified by its chiral vector $\mathbf{C}=$ $(n, m)$, describing the rolling direction in the hexagonal-lattice plane. The resulting spectrum then includes a series of $1 \mathrm{D}$ "cuts" of the 2D Dirac cones, which are determined by the chiral vector [22]. We focus in this work on metallic zigzag nanotubes, i.e., CNTs where $\mathbf{C}=(n, 0)$ and $n \in 3 \mathbb{Z}$, yet our model is easily generalized to any metallic zigzaglike CNT with $n, m \in 3 \mathbb{Z}$ and $n \neq m$, as we discuss below. Importantly, for this kind of CNTs the pure hopping spectrum (without, e.g., SOC) is gapless and fourfold degenerate $(2$ spin $\times 2$ valley) near $k_{\|}=0$, where $k_{\|}$is the momentum along the nanotube axis.

The CNT is modeled by the following tight-binding Hamiltonian on a honeycomb lattice with periodic boundary conditions in a direction determined by $\mathbf{C}$,

$$
\begin{aligned}
H_{\mathrm{CNT}}= & \sum_{i, s, s^{\prime}} c_{i, s}^{\dagger}\left(-\delta^{s s^{\prime}} \mu\left(\theta_{i}\right)-\sigma_{z}^{s s^{\prime}} V_{\mathrm{Z}}\right) c_{i, s^{\prime}} \\
& +\sum_{\langle i, j\rangle, s, s^{\prime}}\left[c_{i, s}^{\dagger}\left(-t \delta^{s s^{\prime}} e^{i A_{i j}}+i \Delta_{o, i j}^{\mathrm{SO}} \sigma_{z}^{s s^{\prime}}\right) c_{j, s^{\prime}}+\text { H.c. }\right] \\
& +\sum_{\langle\langle i, j\rangle\rangle, s, s^{\prime}}\left[i \Delta_{z, i j}^{\mathrm{SO}} c_{i, s}^{\dagger}\left(\sigma_{z}\right)_{s s^{\prime}} c_{j, s^{\prime}}+\text { H.c. }\right] .
\end{aligned}
$$

Here $c_{i, s}$ are creation operators of electrons at the lattice site $i$ with spin $s, t$ is the nearest-neighbor hopping amplitude, $\mu$ is the on-site chemical potential, which in general depends on (a)

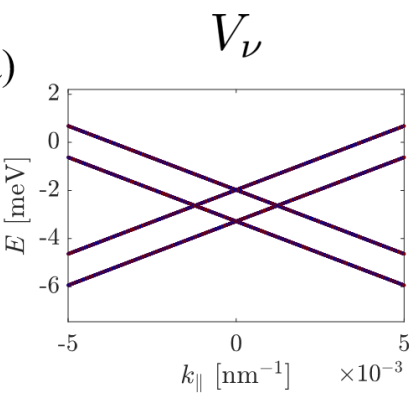

(e)

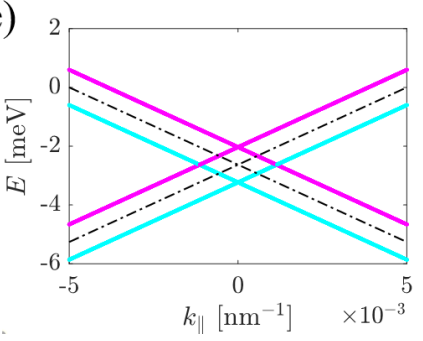

(b)

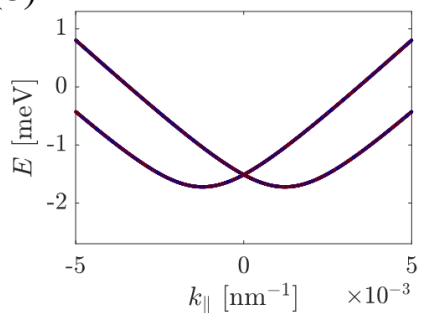

(f)

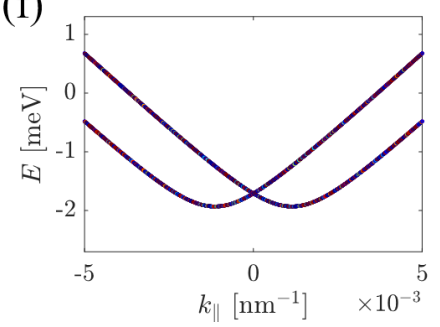

$\phi$

(c)

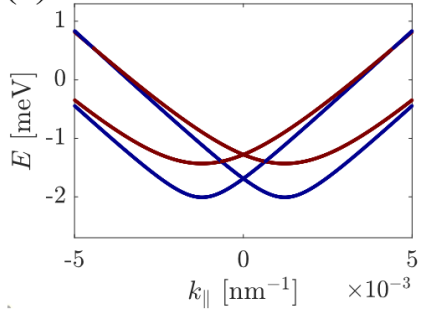

(g)

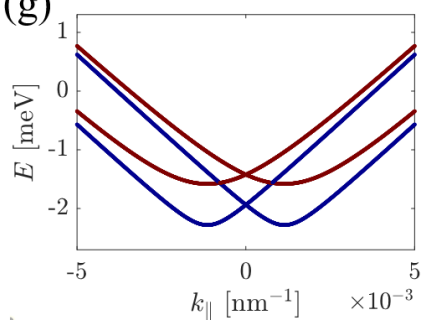

(d)

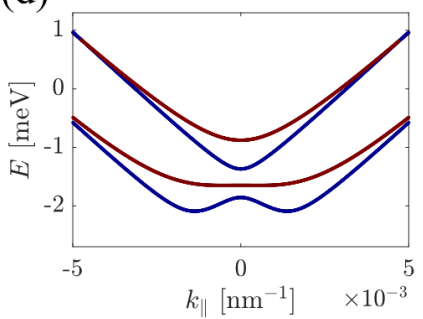

(h)

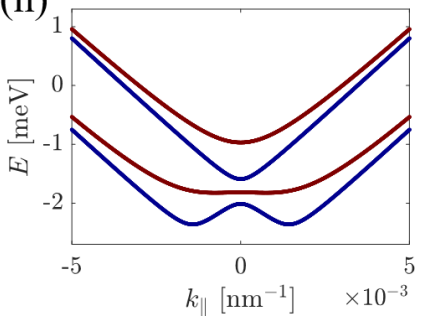

FIG. 2. Spectra of a $(12,0)$ zigzag CNT calculated using the tight-binding Hamiltonian Eq. (1) (top row), and using the effective continuum model Eq. (2) (bottom row). Going from left to right, we consecutively "switch on" different terms in the Hamiltonian. (a) Calculated spectrum with zero magnetic flux and zero SOC terms. The Dirac cone splitting is due to a rotational symmetry breaking by the on-site chemical potential $\mu\left(\theta_{i}\right)=\frac{\mu_{0}}{\sqrt{2 \pi} \Sigma} e^{-\frac{1}{2}\left(\frac{\theta_{i}}{\Sigma}\right)^{2}}$, with $\mu_{0}=40 \mathrm{meV}$, and $\Sigma=0.6$. (b) Same as (a), but with a finite orbital SOC energy $\Delta_{o}^{\text {SO }}=0.7 \mathrm{meV}$. (c) Same as (b), with an added magnetic flux induced by a magnetic field $B_{z}=1 \mathrm{~T}$ applied along the CNT axis. (d) Same as (c), with a finite Zeeman-type SOC $\Delta_{Z}^{\text {SO }}=0.5 \mathrm{meV}$. The plots (e)-(h) correspond to the effective continuum Hamiltonian of plots (a)-(d), respectively. The roles of rotational-symmetry breaking, orbital SOC, Zeeman SOC, and magnetic flux are captured by the parameters $V_{v}=0.6$ meV, $v_{\mathrm{F}} \alpha=0.7 \mathrm{meV}, v_{\mathrm{F}} \alpha^{\prime}=0.5 \mathrm{meV}$, and $v_{\mathrm{F}} \phi=0.35 \mathrm{meV}$, respectively. In (e) the different colors mark the different expectation values of the valley bonding and antibonding $\left\langle v_{x}\right\rangle= \pm 1$ in the two bands. In all other plots red and blue mark the spin projections of the different bands along the nanotube axis, $\sigma= \pm 1$. The tight-binding model and the continuum model agree well, and as expected deviations start to appear when moving away from $k_{\|}=0$. 
the angle $\theta_{i}$ along the CNT's circumference at which the site $i$ is situated, and $\sigma_{z}$ is a Pauli matrix acting in spin space. The magnetic field applied along the CNT axis gives rise to the Zeeman splitting $V_{\mathrm{Z}}$ and to an orbital effect, captured by the Peierls phase $A_{i j}$ [23]. The SOC is accounted for by two terms $\Delta_{o, i j}^{\mathrm{SO}}, \Delta_{z, i j}^{\mathrm{SO}}$, which are the orbital- and Zeeman-type SOC matrix elements between sites $i, j$. The labels $\langle i, j\rangle$ and $\langle\langle i, j\rangle\rangle$ indicate summation over nearest and next-nearest neighbors, respectively. The on-site potential $\mu\left(\theta_{i}\right)$ breaks the azimuthal symmetry of the CNT, enabling intervalley scattering, which will prove crucial for our subsequent analysis. More details regarding the tight-binding model Eq. (1) are given in Appendix A.

We find that the low-energy properties of the tight-binding model Eq. (1) can be approximated by the following continuum model (we set $\hbar=1$ henceforth):

$$
H=v_{\mathrm{F}}\left[\rho_{y} k_{\|}+\rho_{x} v_{z}\left(\alpha \sigma_{z}+\phi\right)+\alpha^{\prime} \sigma_{z} v_{z}\right]+V_{\nu} v_{x}
$$

with $\rho_{i}, \sigma_{i}, v_{i}$ Pauli matrices acting in the subspaces of the sublattice, spin, and valley degrees of freedom, respectively. The Fermi velocity $v_{\mathrm{F}}$ characterizes the linear dispersion near the graphene Dirac cones, with a value of $\sim 8 \times 10^{5} \mathrm{~m} / \mathrm{s}$. The spin-orbit term $\alpha$ corresponds to a spin-dependent phase accumulated by an electron going around the tube's diameter [24]. Its strength is inversely proportional to the diameter of the nanotube [25] and can be roughly estimated [26] as $v_{\mathrm{F}} \alpha \approx 1 \frac{\mathrm{meV}}{R[\mathrm{~nm}]}$, with $R$ the CNT's radius. The strength of the sublattice diagonal "Zeeman-like" SOC term $\alpha$ ' depends on the chirality of the CNT [27], and is usually estimated to be of the same order of magnitude as $\alpha[16,27,28]$. The circumferential momentum shift due to the Aharonov-Bohm
(AB) flux $\phi$ can be written in terms of $R$ and the magnetic field $B_{z}, v_{\mathrm{F}} \phi \approx R[\mathrm{~nm}] B_{z}[\mathrm{~T}] \mathrm{meV}$.

Finally, $V_{v}$, which is responsible for intervalley scattering due to breaking of the CNT rotational symmetry about its axis, caused by, e.g., an anisotropic gate inducing angle-dependent chemical potential, is approximated from our tight-binding analysis to be of order $\sim 1 \mathrm{meV}$, consistent with previous estimates [17]. In Eq. (2) the Zeeman term induced by the external field $B_{z}$ was neglected, as it is small in comparison to the other energy scales for the moderate-to-low magnetic field regime we are interested in (few Tesla or lower). For example, with a magnetic field $B_{z}=1 \mathrm{~T}$ we have $V_{Z} \approx$ $0.1 \mathrm{meV}$, whereas the energy associated with the flux for $R=1 \mathrm{~nm}$ is $V_{\phi}=v_{\mathrm{F}} \phi \approx 0.85 \mathrm{meV}$. For further details on numerically estimating the parameters appearing in Eq. (2) in terms of experimental parameters, see Appendix B. We note that due to the negligible Zeeman energy, and the fact that our proposal relies only on the orbital effect of the magnetic field, the proposed scheme is quite insensitive to deviations in the orientation of the magnetic field, provided that enough flux is threaded through the CNT.

It is instructive to define an antiunitary time-reversal operator $\mathcal{T}=v_{x} \sigma_{y} \mathcal{K}$, with $\mathcal{K}$ the complex conjugation operator, such that $\mathcal{T}^{2}=-1$. Neglecting the Zeeman term, only the $A B$ flux term breaks the time-reversal symmetry, since $H(\phi=0)$ commutes with $\mathcal{T}$, as one would expect. Also notice that in the Hamiltonian Eq. (2) the spin projection along the CNT axis $\sigma_{z}$, is a good quantum number, to be labeled as $\sigma= \pm 1$.

The role of each of the components of $H$ is illustrated in Fig. 2. The low-energy continuum Hamiltonian is readily diagonalized, and we obtain the eigenenergies $E= \pm \epsilon\left(k_{\|}\right)$, with

$$
\epsilon\left(k_{\|}\right)=\sqrt{k_{\|}^{2}+V_{v}^{2}+(\phi+\sigma \alpha)^{2}+\alpha^{\prime 2} \pm 2 \sqrt{\left(k_{\|} V_{v}\right)^{2}+\alpha^{\prime 2}\left[k_{\|}^{2}+(\phi+\sigma \alpha)^{2}\right]}} .
$$

Several key insights may be inferred from the form of $\epsilon\left(k_{\|}\right)$. First, it is evident that only a combination of the magnetic flux $\phi$ and the SOC $\alpha$ terms may lift the spin degeneracy in the spectrum, which is vital for our half-metallic construction. We also see that $V_{v}$ splits the spectrum into two shifted copies in the $k_{\|}$direction, similar to the effect of Rashba SOC in quantum nanowires. Finally, the role of Zeeman-like SOC $\alpha^{\prime}$ is clearly understood in the vicinity of $k_{\|}=0$, where it lifts the twofold degeneracy in the spectrum, thereby opening a gap. The lifting of this degeneracy is also crucial, otherwise one always ends up with an even number of pairs of Fermi points, regardless of the value of the chemical potential. To achieve an odd number of Fermi points, and hence the possibility of a topological phase, one must thus use a CNT which has a finite $\alpha^{\prime}$ SOC term.

A single-channel half-metallic phase is achieved when the spectrum $\epsilon\left(k_{\|}\right)$is tuned such that an energy window with only two Fermi points exist. However, this is not sufficient to ensure that the CNT is susceptible to proximity-induced superconductivity. The Cooper pair that tunnels from the superconductor typically has a small net momentum, and therefore the sum of the two Fermi momenta should also be small. This cannot be achieved if the two Fermi points belong to the same valley, in which case the total momentum of the pair in the circumferential direction $k_{\perp} \sim 1 / R$ is large. This problem is circumvented in our scheme by introducing a potential that breaks the symmetry around the tube. This symmetry breaking is embodied by the term $V_{v} v_{x}$ in Eq. (2). When the value of this term is comparable to the other terms in the Hamiltonian, an appreciable valley mixing is obtained so that $\left|\left\langle v_{x}\right\rangle\right| \approx 1$ and the two Fermi points have opposite momenta, see Fig. 3. Tuning the chemical potential, such that an additional even number of spin channels is occupied, may also lead to topological superconductivity.

\section{EQUAL-SPIN PROXIMITY EFFECT}

So far we have established the possibility of tuning the CNT into a state where it has an odd number of pairs of Fermi crossing points, by exploiting the intrinsic SOC and the orbital effect of a parallel magnetic field. However, an $s$-wave superconductor proximity coupled to the nanotube 


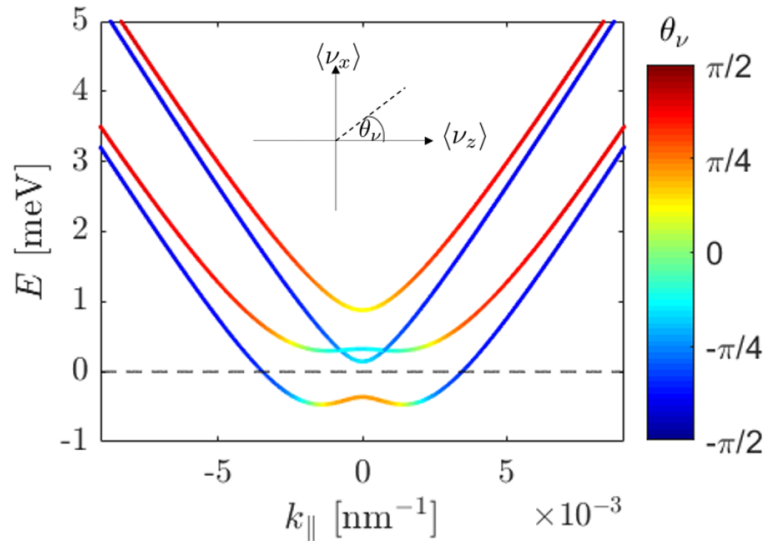

FIG. 3. Zoom-in on part of the CNT spectrum, with the chemical potential tuned to a half-metallic point. The black dashed line marks the Fermi energy, and colors indicate the value of $\theta_{v} \equiv \tan ^{-1} \frac{\left\langle v_{x}\right\rangle}{\left\langle v_{z}\right\rangle}$ (illustrated in the inset); recall that the $v_{i}$ act in the valley subspace, so $\theta_{v}=0$ corresponds to complete valley polarization, which occurs when the rotation-symmetry breaking term $V_{v}$ vanishes and $v_{z}$ is a good quantum number. Here $V_{v}$ is sufficiently large such that the valleys are almost completely mixed near the Fermi points, i.e., $\left|\theta_{\nu}\right| \approx \frac{\pi}{2}$. Parameters used: $v_{\mathrm{F}} \alpha=0.4 \mathrm{meV}, v_{\mathrm{F}} \alpha^{\prime}=0.3 \mathrm{meV}, v_{\mathrm{F}} \phi=$ $2 \mathrm{meV}$, and $V_{v}=1 \mathrm{meV}$.

cannot induce a topological gap, since all the bands are spin polarized. Instead, one needs to use a superconductor which has a significant spin-triplet component, and bring it to contact with the CNT. Moreover, this superconductor should have the right spin-triplet component that will be compatible with the spin polarization of the CNT, which is in the tube axis direction.

We propose the use of superconducting thin films or monolayers of TMDs as a superconducting substrate. In these materials, due to a combination of strong atomic SOC and breaking of the lattice in-plane mirror symmetry, electrons in opposite valleys experience opposite effective Zeeman fields [29,30], an effect known as Ising SOC. Studies of superconducting few-layers TMDs show an increase of the upper critical inplane magnetic field well above the Clogston limit (where the magnetic polarization energy is equal to the superconductor condensation energy) [31-36]. This phenomenon originates in the strong tendency of the electron spins to point in the outof-plane direction due to a strong Ising effective field. It was demonstrated that in the presence of an $s$-wave pairing potential, Ising SOC facilitates equal-spin spin-triplet Cooper pairs, with their spin pointing in the in-plane direction [37]. This scenario is ideal for inducing topological superconductivity in the CNT. Notice that no time-reversal-symmetry breaking within the superconducting TMD needs to occur. Concretely, we suggest the use of one particular material $\mathrm{NbSe}_{2}$, which has an exceptionally high Ising SOC with a spin-splitting energy of about $80 \mathrm{meV}$ in the monolayer [33]. This would ensure that the equal-spin component in the Cooper-pair wave function is comparable with that of the singlet. Moreover, recent experiments with graphene-superconducting $\mathrm{NbSe}_{2}$ heterostructures imply some compatibility between the two, and the possibility of an appreciable proximity effect [38-40], which will presumably also hold true for the CNTs.
We model the proximity-induced pairing terms in the nanotube as

$$
H_{\mathrm{SC}}=\tilde{\Delta}_{s} \sum_{i} \gamma_{i} c_{i \uparrow}^{\dagger} c_{i, \downarrow}^{\dagger}+\tilde{\Delta}_{t} \sum_{\langle i, j\rangle, s=\uparrow, \downarrow} b_{i j} \gamma_{i} \gamma_{j} c_{i, s}^{\dagger} c_{j, s}^{\dagger}+\text { H.c. },
$$

where $\tilde{\Delta}_{s}, \tilde{\Delta}_{t}$ are the singlet and triplet pairing potentials, the indicator $\gamma_{i}$ is 1 if site $i$ lies in the area covered by the SC and 0 otherwise, and $b_{i j}= \pm 1$ depending on the direction of the bond connecting sites $i, j$. The pairing term Eq. (4) can also be captured by the low-energy continuum description. We introduce an antiunitary particle-hole operator $\Lambda=\tau_{y} \mathcal{T}$, with $\tau_{i}$ Pauli matrices acting on the particle-hole degree of freedom (notice that in the tight-binding description the valley degree of freedom is absent, and thus the particle-hole operator is $\left.\Lambda_{\mathrm{TB}}=\tau_{x} \mathcal{K}\right)$. We may now write the continuum Bogoliubovde Gennes (BdG) Hamiltonian,

$$
\begin{aligned}
H_{\mathrm{BdG}}= & \left\{v_{\mathrm{F}}\left[\rho_{y} k_{\|}+\left(\rho_{x} \alpha+\alpha^{\prime}\right) \sigma_{z} v_{z}\right]+V_{v} v_{x}-\mu\right\} \tau_{z} \\
& +v_{\mathrm{F}} \rho_{x} v_{z} \phi+\left(\Delta_{s}+\Delta_{t} \rho_{y} \sigma_{x}\right) \tau_{x},
\end{aligned}
$$

which acts on a Nambu spinor with a total of 16 components (sublattice, spin, valley, and particle hole). $\Delta_{s}, \Delta_{t}$ are the low-energy counterparts of the tight-binding pairing potentials introduced in Eq. (4), $\tilde{\Delta}_{s}, \tilde{\Delta}_{t}$, respectively. The particle-hole symmetry is manifested by $\left\{H_{\mathrm{BdG}}, \Lambda\right\}=0$. The form of the spin-triplet term in $H_{\mathrm{BdG}}$ is not only consistent with the tightbinding simulations, but it is also the only possible pairing term which (i) preserves particle-hole and time-reversal symmetries, (ii) pairs nearest-neighbor equal-spin electrons with zero circumferential momentum, and (iii) does not distinguish between different valleys. Although the applied magnetic field breaks the time-reversal symmetry in our system, the intrinsic pairing in the superconducting substrate does not. The same argument applies with regards to the breaking of the valley symmetry by $V_{\nu}$. Thus, this pairing term, along with the singlet one, is the main focus in this work. We note that in the absence of singlet pairing, $\Delta_{s}=0$, the spin conservation in the system is reflected by $\left[H_{\mathrm{BdG}}, \sigma_{z} \tau_{z}\right]=0$, i.e., $\sigma_{z}$ is no longer a good quantum number, but $\sigma_{z} \tau_{z}$ is. A finite $\Delta_{S}$ breaks this symmetry as it mixes the spins, but is not necessarily detrimental to the emergence of the topological superconducting phase, as we will later show.

A lattice BdG Hamiltonian can be used to diagnose the parameter regimes where topological superconductivity takes place by introducing the $\mathbb{Z}_{2}$ topological index $[2,41,42]$,

$$
Q=\operatorname{sgn}\left[\operatorname{Pf}\left\{\Lambda H_{\mathrm{BdG}}\left(k_{\|}=0\right)\right\} \operatorname{Pf}\left\{\Lambda H_{\mathrm{BdG}}\left(k_{\|}=\pi\right)\right\}\right],
$$

where $\operatorname{Pf}\{\cdot\}$ is the matrix Pfaffian. $Q=-1$ corresponds to the topologically nontrivial phase, whereas in the trivial phase $Q=1$. For the case of a continuum $\mathrm{BdG}$ Hamiltonian as in Eq. (5), the Pfaffian should only be evaluated at $k_{\|}=0$.

\section{EMERGENCE OF TOPOLOGICAL SUPERCONDUCTIVITY}

Now that we have the full BdG Hamiltonian Eq. (5), we may explore the parameter space in order to find the topological phases. As an example, using realistic parameters for the CNT, as well as for the superconducting TMD substrate, we 

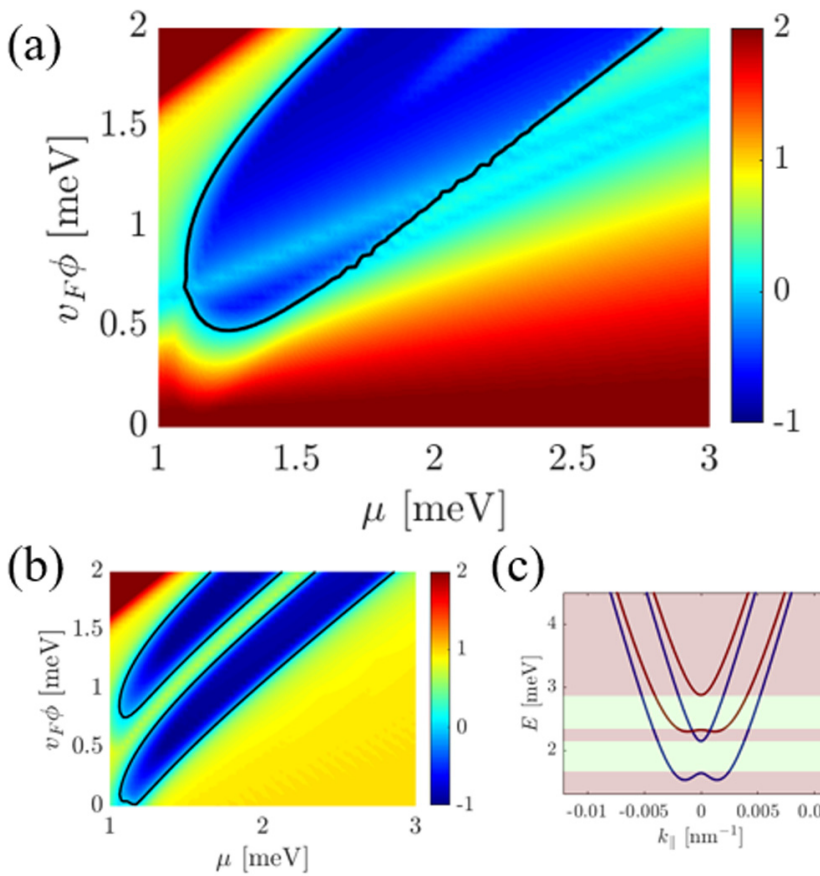

(c)

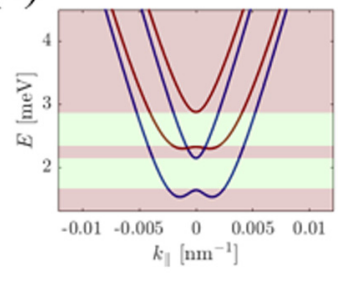

FIG. 4. (a) Phase diagram of the proximity-coupled CNT as a function of the magnetic flux and chemical potential. Shown is the BdG quasiparticle energy gap, normalized by the triplet pairing strength $\frac{E_{\mathrm{g}}}{\Delta_{\mathrm{t}}}$, multiplied by the topological index $Q$. Regions with negative values (blue) are topological. The black lines demarcate the topological phase transitions, where the gap closes. The parameters used are $v_{\mathrm{F}} \alpha=0.4 \mathrm{meV}, v_{\mathrm{F}} \alpha^{\prime}=0.3 \mathrm{meV}, V_{v}=1 \mathrm{meV}$, $\Delta_{t}=0.1 \mathrm{meV}$, and $\Delta_{s}=0.3 \mathrm{meV}$. (b) The same as (a), only with the spin-singlet component of the proximity effect $\Delta_{s}=0$. (c) The normal-state CNT spectrum, with the same parameters as in Fig. 3, with different line colors for the two spin directions. We mark the region with an odd (even) number of Fermi crossing pairs with a bright green (pink) background. The small pink "window" in between the bright green regions corresponds to the trivial phase in between the two topological ones in (b).

show in Fig. 4(a) that by "scanning" the gate voltage and the magnetic flux a large topological area in parameter space is indeed accessible, with a quasiparticle gap $E_{\mathrm{g}}$ comparable in size to the triplet pairing potential $\Delta_{t}$.

Interestingly, while $\Delta_{t}$ is the crucial ingredient for our scheme to produce topological $p$-wave superconductivity, the presence of a finite singlet component $\Delta_{s}$ can be beneficial in some cases. This can be seen by comparing Figs. 4(a) and $4(\mathrm{~b})$, where in the latter $\Delta_{s}=0$, and a trivial regime emerges in the middle of the area which was topological in the former. To understand why, one should examine the number of Fermi level crossings in the normal-state spectrum, see Fig. 4(c). When varying, e.g., the chemical potential, a crossover occurs from an odd number of crossing pairs to an even one. Having only same-spin pairing would thus mean we have a topological phase transition to the trivial phase. A finite $\Delta_{s}$ may however bridge between the two topological phases by allowing more pairing interactions in the intermediate region. Another consequence of finite $\Delta_{s}$ is an increase of the minimal magnetic field required to access the topological phase. This is to be expected,

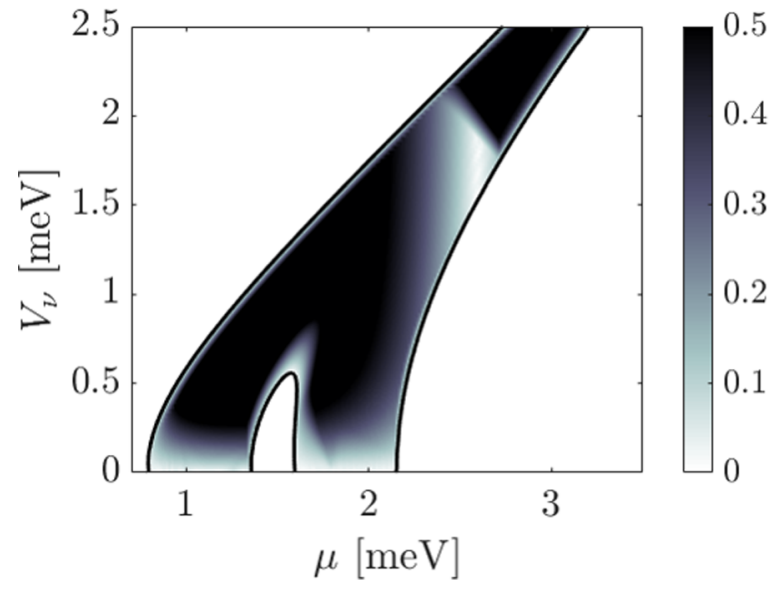

FIG. 5. Normalized quasiparticle energy gap $\frac{E_{\mathrm{g}}}{\Delta_{t}}$, as a function of the chemical potential $\mu$ and the inter-valley mixing strength $V_{v}$. The color scale is such that only the variations inside the topological regime are shown. Notice that the gap vanishes as $V_{v} \rightarrow 0$. The parameters used are $v_{\mathrm{F}} \alpha=0.4 \mathrm{meV}, v_{\mathrm{F}} \alpha^{\prime}=0.3 \mathrm{meV}, v_{\mathrm{F}} \phi=1.5 \mathrm{meV}$, $\Delta_{t}=0.1 \mathrm{meV}$, and $\Delta_{s}=0.3 \mathrm{meV}$.

since $\phi$ is necessary to establish the half-metallic phase, in which there exists a regime where the spin-singlet pairing is ineffective.

Let us comment on the strength of the magnetic field required to tune the system into the topological phase. The SOC parameters chosen for Fig. 4 are appropriate for a CNT of radius $2.5 \mathrm{~nm}$. The minimal $v_{\mathrm{F}} \phi$ required to make this CNT topological is about $0.5 \mathrm{meV}$, which by the relation $B_{z}[T] \approx v_{\mathrm{F}} \phi[\mathrm{meV}] / R[\mathrm{~nm}]$ corresponds to a magnetic field of $200 \mathrm{mT}$ (see Appendix B). In this example we find that the gap is $E_{\mathrm{g}} \approx 0.1 \mathrm{meV}$. The localization length of the Majorana zero mode is $\xi \approx v_{\mathrm{F}} / E_{\mathrm{g}} \approx 5 \mu \mathrm{m}$, and we expect that it may be smaller due to a reduction of the Fermi velocity by the superconductor [43]. Following this consideration we estimate that a nanotube with a radius of $10 \mathrm{~nm}$ requires only about $50 \mathrm{mT}$ to become topologically nontrivial, albeit with a smaller gap.

We can now also better appreciate the role of $V_{v}$ in $H_{\mathrm{BdG}}$. Examining the amplitude of the topological gap as a function of $V_{v}$, see Fig. 5, we see that near $V_{v}=0$ there exists a region which although being formally topological as $Q=$ -1 , has a very small energy gap $E_{\mathrm{g}}$-it is diminished by around one order of magnitude compared to $\Delta_{t}$. This is merely a consequence of the valley polarization in the absence of the rotational-symmetry breaking, which attenuates zero-momentum Cooper-pair hopping into the CNT. Only when the average valley number $\left\langle v_{z}\right\rangle$ approaches zero, namely the electron wave functions at the Fermi surface are evenly distributed between $K$ and $K^{\prime}$, can the full superconducting gap develop in the system, as is the case for larger values of $V_{v}$.

\section{MAJORANA EDGE STATES}

We now turn to demonstrate the topological phase transition from real-space diagonalization of the tight-binding Hamiltonian Eqs. (1) and (4). To this end we simulate a 

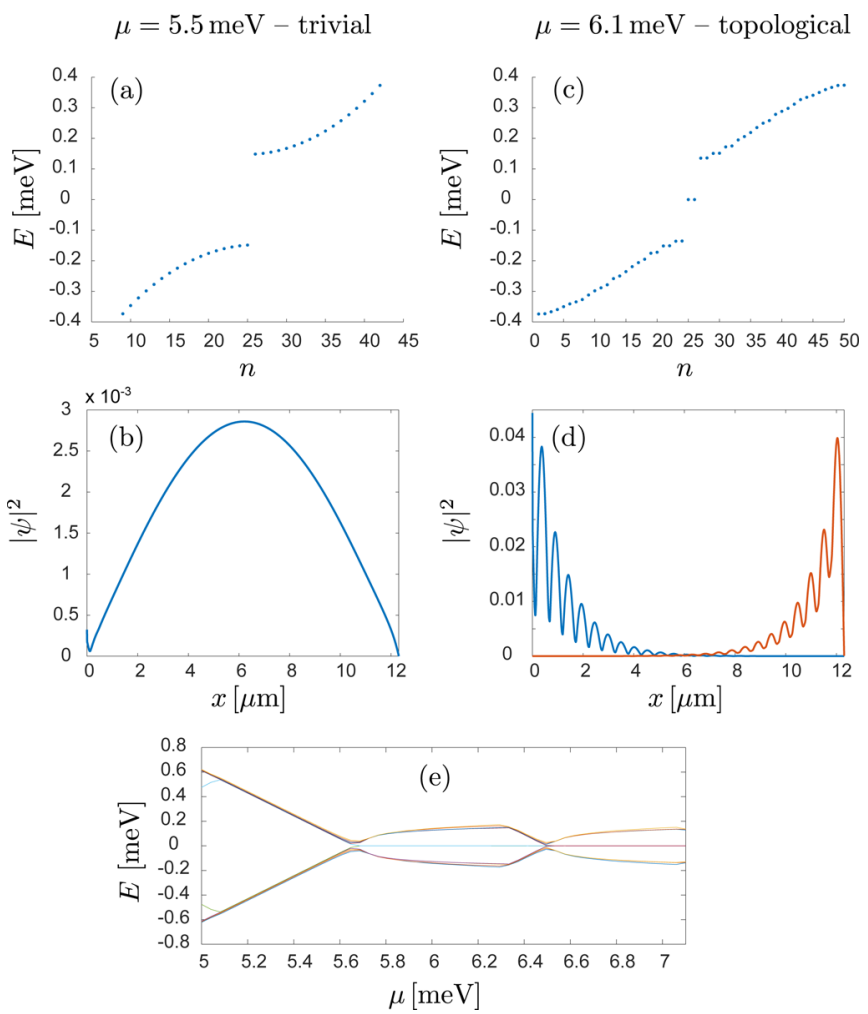

FIG. 6. Topologically trivial (a) and (b) and nontrivial (c) and (d) phases of the proximitized CNT. The trivial phase is gapped and all of its eigenstates are bulk states. In contrast, the topological phase exhibits a pair of zero-energy modes within the bulk gap, and their wave functions are localized at the CNT's edges. The spectra are shown in (a) and (c); in (b) and (d) we show the absolute value squared of the electronic wave functions. The two curves in (d) are obtained as follows: Starting from two degenerate zero-energy eigenstates, we multiply one of them by a global phase so its first component matches the other's, and then we take the symmetric and antisymmetric combination of the resulting wave functions. This yields two states localized at opposite edges of the CNT; a tunneling density of states measurement would produce the sum of the two curves displayed. (e) Ordered eigenenergies (absolute value squared) as a function of the chemical potential $\mu$. The topological phase transitions are observed as gap closings and re-openings, where in the topological phase zero-energy modes persist inside the bulk gap.

finite-length CNT with open boundary conditions. We exemplify our results on a $(6,0)$ CNT of length $12.3 \mu \mathrm{m}$ $\left(1.2 \times 10^{6}\right.$ carbon atoms). We use the realistic parameters $t=$ $2.66 \mathrm{eV}$ [44], $\Delta_{o}^{\mathrm{SO}}=2 \mathrm{meV}, \Delta_{z}^{\mathrm{SO}}=1 \mathrm{meV}$ [26], and assume an intervalley mixing energy of $15 \mathrm{meV}$ in a steplike structure (see Appendix A for details). A modest magnetic field of $2 \mathrm{~T}$ is used in order to drive the system into the topological phase (for CNTs of larger diameter, even a weaker magnetic field will suffice). For simplicity, we discard the spin-singlet component of the SC $\tilde{\Delta}_{s}=0$, and only include a spin-triplet component $\tilde{\Delta}_{t}=0.5 \mathrm{meV}$. Furthermore, we present results for a CNT fully covered by a SC; we have tested also the case of partial covering and saw almost no difference, apart from a suppression of the energy gap due to the smaller effective $\Delta_{t}$ experienced by the CNT.
The phase transition may be observed in Fig. 6, where we show the BdG spectrum and the lowest-energy wave function for the trivial and topological phases (we control the crossover by tuning the chemical potential $\mu$ ). The trivial phase is gapped and has no edge modes, whereas the topological phase exhibits two zero-energy modes localized at the edges of the CNT. The Majorana localization length can be roughly estimated as $\xi_{\mathrm{M}}=v_{\mathrm{F}} / E_{\mathrm{g}}$, which is of the order of $1 \mu \mathrm{m}$.

Another way to observe the topological phase transition is inspecting the BdG spectrum as a function of one of the parameters, e.g., $\mu$, see Fig. 6(e). The topological phase transitions are signaled by closings of the bulk gap. The gap then re-opens inside the phases, but in the topological phase, zero-energy modes clearly appear inside the gap.

\section{TIME-REVERSAL-INVARIANT TOPOLOGICAL SUPERCONDUCTIVITY}

The combination of a tunable CNT and a superconducting TMD substrate may give rise to time-reversal-invariant topological superconductivity in the absence of any magnetic field. This is only made possible in the presence of strong enough electron-electron interactions [45], which heavily suppress the proximity induced spin-singlet component of the superconductivity as compared to the spin-triplet one. Then, a timereversal-invariant topological superconducting phase $[21,46]$ manifests itself in the system.

We now consider thin CNTs, in which interactions play a more significant role [47], and that have a substantial curvature-induced gap, such as zigzag CNTs $(3 n, 0)$, with an integer $n$. We note that this gap scales as $1 / R^{2}$ for zigzaglike CNTs [48], which is the reason it was neglected in previous sections (where mainly large-diameter CNTs were considered). The noninteracting part of the Hamiltonian is described by the low-energy theory

$$
H_{0}=v_{\mathrm{F}}\left[k \rho_{y}+\left(\kappa+\alpha v_{z} \sigma_{z}\right) \rho_{x}+\alpha^{\prime} v_{z} \sigma_{z}\right]+V_{v} v_{x}-\mu,
$$

and $v_{\mathrm{F}} \kappa$ plays the role of the curvature gap. When $H_{0}$ is dominated by $\kappa$, the spectrum rather simplifies, see Figs. 7(a) and 7(b). Spin degeneracy is not lifted (since no magnetic flux is introduced), and the different bands are approximate $v_{x}$ eigenstates, slightly modified by the presence of spin-orbitcoupling terms.

By properly adjusting a gate voltage, and thus $\mu$, one can tune to a point where the Fermi level crosses a single spin-degenerate band. At this level we effectively describe our system as a 1D system with two spin species. This system is proximity coupled to a superconductor having a spin-singlet component $\Delta_{s}$, as well as a spin-triplet component $\Delta_{t}$.

We note that the CNT origin of this effective Hamiltonian should not be entirely cast away. For example, upon adding the BdG term $\Delta_{t}^{0} \rho_{y} \sigma_{x} \tau_{x}$ and examining the energy spectrum, we find that the curvature term $\kappa$ reduces the pairing gap to $\Delta_{t} \approx \Delta_{t}^{0} \sqrt{\frac{k_{F}^{2}}{k_{F}^{2}+\kappa^{2}}}$. 
(a)

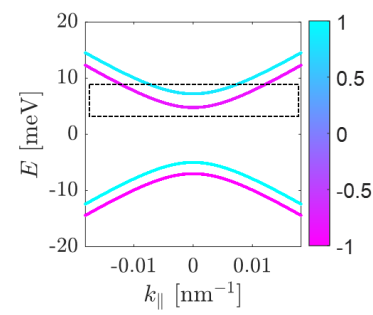

(c)

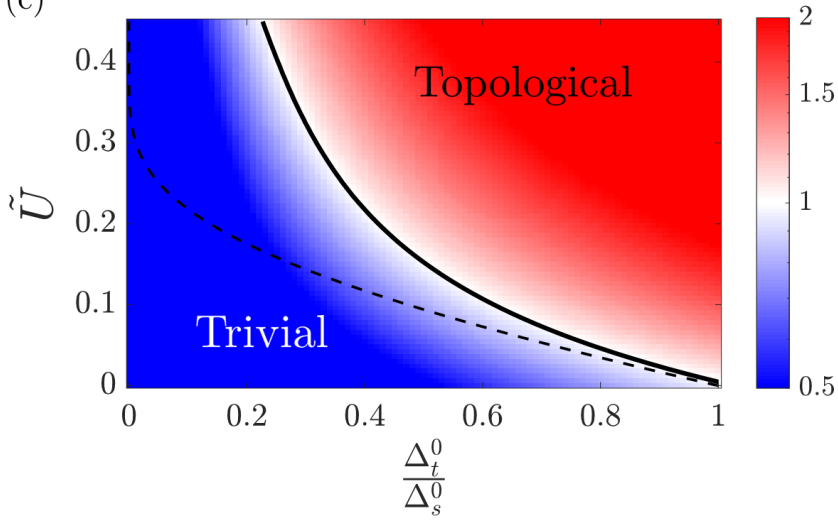

FIG. 7. (a) Normal-state spectrum of the CNT with no magnetic field and with a finite curvature-induced gap. The color scale indicates $\left\langle v_{x}\right\rangle$. All bands are exactly spin degenerate. Parameters used here are $v_{\mathrm{F}} \alpha=0.4 \mathrm{meV}, v_{\mathrm{F}} \alpha^{\prime}=0.3 \mathrm{meV}, V_{v}=1 \mathrm{meV}$, and $v_{\mathrm{F}} \kappa=6 \mathrm{meV}$. (b) Zoom-in on a small part of the spectrum marked by dashed lines in (a). (c) Phase diagram of the spin-degenerate system with interactions, in the presence of the two types of proximity induced superconducting pairing. As a function of initial conditions, we plot the ratio $\frac{\Delta_{t}\left(\ell^{*}\right)}{\Delta_{s}\left(\ell^{*}\right)}$, with $\ell^{*}$ the RG time at which the first of the pairing potentials reaches strong coupling. The solid black line marks the transition $\frac{\Delta_{t}\left(\ell^{*}\right)}{\Delta_{s}\left(\ell^{*}\right)}=1$. The dashed black line marks the same transition, calculated using the approximate phase boundary Eq. (10). As expected, this approximation overestimates the prominence of the topological sector. Notice that the color scale here is logarithmic, and that we used the single parameter $\tilde{U}$ to account for all interactions. For this plot we used the initial condition $\delta_{s}^{0}=0.05$. The definition of this parameter, as well as the full RG equations, are all found in Appendix C.

Upon linearization of the spectrum near the Fermi points, we write the Hamiltonian density

$$
\begin{aligned}
\mathcal{H}= & \sum_{\sigma, r} \psi_{r \sigma}^{\dagger}\left(i r v_{\mathrm{F}} \partial_{x}-\mu\right) \psi_{r \sigma}+\mathcal{H}_{\text {int }} \\
& +\left[\Delta_{s}\left(\psi_{R \uparrow} \psi_{L \downarrow}+\psi_{L \uparrow} \psi_{R \downarrow}\right)+\text { H.c. }\right] \\
& +\left[\Delta_{t}\left(\psi_{R \uparrow} \psi_{L \uparrow}-\psi_{R \downarrow} \psi_{L \downarrow}\right)+\text { H.c. }\right],
\end{aligned}
$$

where $\psi_{r \sigma}$ annihilates a fermion with spin $\sigma$ and chirality $r=R, L$, and $\mathcal{H}_{\text {int }}$ accounts for interactions. The form of the pairing $\Delta_{t}$ is dictated by the spin polarization of the electrons in the CNT along the tube axis, the Ising nature of the TMD with out-of-plane spin polarization, and time-reversal symmetry. The relative minus sign between the two-species spin-triplet proximity term ensures that for $\Delta_{s}=0$ the system is in the topological phase [20]. To get a transition into a trivial phase, the BdG gap must be closed. For the noninteracting
Hamiltonian this occurs when $\left|\Delta_{s}\right|=\left|\Delta_{t}\right|$, hence we have the topological condition

$$
\left|\Delta_{S}\right|<\left|\Delta_{t}\right| \text {. }
$$

One generically expects the singlet proximity component to be greater (even if comparable in size) to the spin-triplet one. We find, however, that although this may indeed be true for the bare values of the superconducting gaps, interactions renormalize both proximity terms. This renormalization naturally favors the triplet over the singlet component, as we show in Appendix C.

More concretely, we find at the tree level of the renormalization group (RG) flow that the topological condition Eq. (9) is modified by accounting for interactions into

$$
\Delta_{t, 0} / \Lambda \geqslant\left(\Delta_{s, 0} / \Lambda\right)^{\frac{4-K_{c}^{-1}-K_{s}^{-1}}{4-K_{c}^{-1}-K_{s}-y}},
$$

with $\Delta_{s / t, 0}$ the bare proximity terms, $K_{c}$ and $K_{s}$ are the Luttinger parameters of the charge and spin sectors, respectively, $\Lambda$ is the energy cutoff of our theory, and $y$ is a dimensionless coupling accounting for backscattering interactions (see Appendix $\mathrm{C}$ for more details). For generic repulsive interactions, one has the bare values

$$
K_{c}^{0}<1, \quad K_{s}^{0}>1, \quad y^{0}>0,
$$

and thus it is becomes clear from Eq. (10) that repulsive interactions enhance the topological part of the phase diagram. For small Hubbard-like interactions characterized by a single dimensionless parameter $\tilde{U}>0$, Eq. (10) can be written in a simpler form $\Delta_{t, 0} / \Lambda \geqslant\left(\Delta_{s, 0} / \Lambda\right)^{\frac{1}{1-2 \tilde{U}}}$. The interactiondependent phase boundary gets distorted by the higher-order corrections to the RG flow, which tend to favor the trivial phase.

We integrate the full RG equations derived in Appendix C, up to the point where one of the pairing potentials reaches strong coupling. At this point, the ratio between the two $\Delta$ 's is extracted, and it is plotted in Fig. 7(c). The main observation is the fact that even if $\Delta_{s}$ is initially significantly larger than $\Delta_{t}$, as is presumably the case when proximitizing the system to a superconducting TMD, strong enough interactions drive the system to a time-reversal-invariant topological superconducting phase.

We finally comment on the departure from the Hubbardlike interactions. Longer-range interactions will have a threefold effect on our equations: they will make $y$ smaller, drive $K_{s}$ much closer to 1 , and significantly decrease the value of $K_{c}$. For a given $K_{c}$ the first two of these effects will obviously favor the singlet pairing, and reduce the topological area in parameter space. However, for an even smaller $K_{c}$, there is a possibility that $\Delta_{s}$ becomes irrelevant, whereas $\Delta_{t}$ is still (perhaps barely) relevant. Thus, long-range interactions do not necessarily eliminate the topological phase from the phase diagram, although one does expect to find a smaller topological gap in this case.

\section{CONCLUSIONS}

In this work we have presented a scheme for realizing robust 1D topological superconductivity in an accessible experimental platform-CNTs, which are very clean and have 
true 1D properties. By applying a magnetic field parallel to the axis of the CNT, and exploiting an orbital effect instead of Zeeman splitting, we were able to demonstrate the emergence of the topological phase at relatively low magnetic fields, compared to previous suggestions [17].

The proposed scheme also requires the use of a superconductor with a spin-triplet component as the reservoir of electron pairs for our system. The use of superconducting monolayer Ising type TMDs, such as $\mathrm{NbSe}_{2}$, is proposed, due to the unusually strong out-of-plane spin-locking characteristic of their charge carriers. This in turn ensures that a significant spin-triplet component exists in the superconducting wave function, which may then couple to the half-metallic CNT. Using monolayer TMDs allows some control of the chemical potential, and also ensures that superconductivity is preserved when a moderate magnetic field is applied. Other suitable candidates for a superconducting substrate with a sizable spin-triplet component are monolayer $\mathrm{TaS}_{2}$ [35], gatetuned $\mathrm{WTe}_{2}$ [49] and $\mathrm{MoS}_{2}$ [31,50], and ultrathin layers of $\mathrm{Pb}$ [51]. A promising alternative proximitizing substrate is magic-angle twisted bilayer graphene, which was recently discovered to support a superconducting phase [52]. It was suggested that significant interaction effects may favor spintriplet superconductivity in this system [53], making it adequate for our scheme.

In fact, we have shown that half-metallicity is not strictly necessary, since a "one-and-a-half" metallic state can be made topologically superconducting due to an odd number (three) of topologically gapped channels. Moreover, in certain cases a residual spin-singlet component of the superconductor may help drive an otherwise trivial regime, i.e., where the normal-state spectrum has an even number of channels, to a topological one, provided that $\Delta_{s}$ is not too large compared to the spin splitting (induced by the magnetic flux and SOC).

In addition, we analyzed the fate of the system in the absence of a magnetic field. We found that the presence of a spin-triplet component in the superconducting proximitizing substrate can make a time-reversal-invariant topological phase accessible. Since interactions tend to decrease the amplitude of spin-singlet pairing more than spin-triplet pairing, one may end up in a state with a Majorana-Kramers pair of zero modes, protected by time-reversal symmetry. Our analysis of the Coulomb-interaction effects in the CNT on the induced pairing have important consequences for the finite-flux case as well. Namely, they suggest a generic suppression of the spin-singlet component as compared to the triplet one. This in turn may enable us to access the topological phase with an even smaller applied magnetic field, see Figs. 4(a) and 4(b).

To examine the real-space wave function, the numerical values of the gaps, and the finite-size effects we have simulated numerically a thin $(6,0) \mathrm{CNT}$, with $1.2 \times 10^{6}$ carbonatom sites, and found a good agreement with the low-energy Hamiltonian Eq. (5). Using the low-energy description, with physical parameters of thicker CNTs (given in Appendix B) we find that the magnetic field required to tune them into the topological phase is much smaller than the one we simulated. For example, for a CNT of radius $2.5 \mathrm{~nm}$ on top of $\mathrm{NbSe}_{2}$ our consideration gives a topological superconductor at $200 \mathrm{mT}$ with a gap of order $0.1 \mathrm{meV}$. A thicker CNT requires an even smaller field to become topological, but the gap is expected to be smaller.

\section{ACKNOWLEDGMENTS}

We thank Erez Berg and Ady Stern for insightful discussions. This work was partially supported by ISF Research Grant in Quantum Technologies and Science, Israel Science Foundation (2074/19).

O.L. and G.S. contributed equally to this work.

\section{APPENDIX A: DETAILS OF THE TIGHT-BINDING SIMULATIONS}

Here we provide some technical details regarding the implementation of the tight-binding model. First, let us explicitly formulate the Peierls phase [23] associated with the magnetic flux $\Phi$ through the tube. Labeling the location of site $i$ in the graphene lattice $\mathbf{r}_{i}$, the Peierls phase is given by [54]

$$
A_{i j}=2 \pi \frac{\Phi}{\Phi_{0}} \frac{\left(\mathbf{r}_{i}-\mathbf{r}_{j}\right) \cdot \mathbf{C}}{|\mathbf{C}|^{2}},
$$

where $\Phi_{0}=h / e$ is the magnetic flux quantum. The SOC terms are essentially spin-dependent hopping terms, and are thus given by [54]

$$
\Delta_{o / z, i j}^{\mathrm{SO}}=\Delta_{o / z}^{\mathrm{SO}}\left(\frac{\left(\mathbf{r}_{i}-\mathbf{r}_{j}\right) \cdot \mathbf{C}_{h}}{\left|\mathbf{r}_{i}-\mathbf{r}_{j}\right| \cdot\left|\mathbf{C}_{h}\right|}\right)^{2} \operatorname{sgn}\left[\left(\mathbf{r}_{i}-\mathbf{r}_{j}\right) \cdot \mathbf{C}_{h}\right],
$$

where $\Delta_{o / z}^{\mathrm{SO}}$ is the "bare" orbital/Zeeman SOC strength.

The intervalley mixing term $V_{v}$ in the low-energy Hamiltonian Eq. (2) corresponds to the angle-dependent potential $V(\theta)$ in the tight-binding description Eq. (1). To implement this term, we first calculate the angle of each site along the CNT's circumference $\theta_{i}=2 \pi \mathbf{r}_{i} \cdot \mathbf{C} /|\mathbf{C}|^{2}$. We examined several forms for the function $V(\theta)$ which is aimed at mimicking the effect of the gate potential, and they all yielded similar results. One possible form is a steplike structure,

$$
V_{\text {step }}(\theta)= \begin{cases}V_{0}, & \theta_{1} \leqslant \theta \leqslant \theta_{2}, \\ 0, & \text { otherwise. }\end{cases}
$$

Another reasonable form is a Gaussian potential,

$$
V_{\text {Gaussian }}(\theta)=V_{0} \exp \left[-\frac{\left(\theta-\theta_{0}\right)^{2}}{(\Delta \theta)^{2}}\right]
$$

In our simulations we mostly used $V_{\text {step }}(\theta)$ with $\theta_{1}=0, \theta_{2}=$ $\pi$, i.e., the voltage at half of the CNT is shifted, thus breaking the azimuthal symmetry.

\section{APPENDIX B: EXPERIMENTAL PARAMETERS}

We bring here for convenience explicit expressions that relate the effective low-energy parameters to experimental parameters of the CNT. The chiral vector $\mathbf{C}=(n, m)$ of the $\mathrm{CNT}$ is related to its radius by

$$
R=\frac{a|\mathbf{C}|}{2 \pi},
$$

with the unit cell size $a=\sqrt{3} a_{\mathrm{CC}} \approx 0.25 \mathrm{~nm}\left(a_{\mathrm{CC}}\right.$ is the separation between nearest-neighbor carbon atoms), and $|\mathbf{C}|=$ $\sqrt{n^{2}+n m+m^{2}}$. 
The magnetic flux term $v_{\mathrm{F}} \phi=\hbar v_{\mathrm{F}} \frac{1}{R} \frac{\Phi}{\Phi_{0}}$, with $\Phi_{0}=2 \times$ $10^{-15} \mathrm{~T} \mathrm{~m}^{2}$ the flux quantum, and $\Phi=\pi R^{2} B_{z}$ the flux through the CNT. The Fermi velocity of the Dirac cones is estimated as $v_{\mathrm{F}} \approx 10^{6} \mathrm{~m} / \mathrm{s}$. We thus find

$$
v_{\mathrm{F}} \phi \approx B_{z}[\mathrm{~T}] R[\mathrm{~nm}] \mathrm{meV} \approx \frac{|\mathbf{C}|}{24} B_{z}[\mathrm{~T}] \mathrm{meV} .
$$

The spin-orbit-coupling term $v_{\mathrm{F}} \alpha$ can be estimated from previous studies [26] which found the spin-orbit gap $\Delta_{\mathrm{SO}} \approx$ $0.4 \mathrm{meV}$ for CNT with radius of $2.5 \mathrm{~nm}$, and thus

$$
v_{\mathrm{F}} \alpha \approx 1 \frac{\mathrm{meV}}{R[\mathrm{~nm}]} \approx \frac{25}{|\mathbf{C}|} \mathrm{meV} .
$$

For reference, we bring here also the Zeeman energy $V_{\mathrm{Z}}=$ $g \mu_{\mathrm{B}} B_{z}$, with $g \approx 2$ and $\mu_{\mathrm{B}}$ the Bohr magneton,

$$
V_{\mathrm{Z}} \approx 0.11 B[\mathrm{~T}] \mathrm{meV} \text {. }
$$

\section{APPENDIX C: INTERACTIONS IN THE TIME-REVERSAL INVARIANT CASE}

To account for the important effect of electron-electron interactions introduced in Sec. VI, we bosonize the Hamiltonian Eq. (8) using standard identities $\psi_{r \sigma} \sim \frac{1}{\sqrt{2 \pi a}} e^{-i\left(r \phi_{\sigma}-\theta_{\sigma}\right)}$, with $a$ some short-distance cutoff, and the bosonic fields satisfying the algebra $\left[\phi_{\sigma}(x), \partial_{x} \theta_{\sigma^{\prime}}\left(x^{\prime}\right)\right]=i \pi \delta_{\alpha \beta} \delta\left(x-x^{\prime}\right)$ [55]. By defining the charge and spin sectors $\phi_{c, s} \equiv \frac{\phi_{\uparrow} \pm \phi_{\downarrow}}{\sqrt{2}}$, this representation allows us to reorganize our Hamiltonian into four parts, $\mathcal{H}=\mathcal{H}_{c}+\mathcal{H}_{s}+\mathcal{H}_{\Delta_{s}}+\mathcal{H}_{\Delta_{t}}$, with

$$
\begin{aligned}
& \mathcal{H}_{c}=\frac{v_{c}}{2 \pi}\left[K_{c}^{-1}\left(\partial_{x} \phi_{c}\right)^{2}+K_{c}\left(\partial_{x} \theta_{c}\right)^{2}\right], \\
& \mathcal{H}_{s}=\frac{v_{s}}{2 \pi}\left[K_{s}^{-1}\left(\partial_{x} \phi_{s}\right)^{2}+K_{s}\left(\partial_{x} \theta_{s}\right)^{2}\right]+\frac{g}{2 \pi^{2} a^{2}} \cos \left(\sqrt{8} \phi_{s}\right),
\end{aligned}
$$

$\mathcal{H}_{\Delta_{s}}=\frac{2 \Delta_{s}}{\pi a} \cos \left(\sqrt{2} \theta_{c}\right) \cos \left(\sqrt{2} \phi_{s}\right)$,

$\mathcal{H}_{\Delta_{t}}=\frac{2 \Delta_{t}}{\pi a} \cos \left(\sqrt{2} \theta_{c}\right) \cos \left(\sqrt{2} \theta_{s}\right)$, with the interaction incorporated into the so-called Luttinger parameters $K_{\eta}, v_{\eta}(\eta=c, s)$, and into the backscattering term $g$ [56]. For our purposes we will approximate $v_{c} \approx v_{s} \equiv v$. This representation makes clear the competition between the two pairing terms, as well as between the backscattering $g$ and $\Delta_{s}$, whose energy cannot be simultaneously minimized for any $g>0$.

Defining the dimensionless constants $y=\frac{g}{\pi v}, \delta_{s / t}=$ $\frac{4 \Delta_{s / t} a}{v} \equiv \frac{\Delta_{s / t}}{\Lambda}$, the RG equations may be derived in a straightforward manner [57],

$$
\begin{aligned}
\frac{d}{d \ell} y & =\left(2-2 K_{s}\right) y-\frac{1}{4} \delta_{s}^{2}, \\
\frac{d}{d \ell} \delta_{s} & =\frac{1}{2}\left(4-K_{c}^{-1}-K_{s}-y\right) \delta_{s}, \\
\frac{d}{d \ell} \delta_{t} & =\frac{1}{2}\left(4-K_{c}^{-1}-K_{s}^{-1}\right) \delta_{t}, \\
\frac{d}{d \ell} K_{s} & =-\frac{1}{2} K_{s}^{2}\left(y^{2}+\frac{1}{4} \delta_{s}^{2}\right)+\frac{1}{8} \delta_{t}^{2}, \\
\frac{d}{d \ell} K_{c} & =\frac{1}{8}\left(\delta_{t}^{2}+\delta_{s}^{2}\right) .
\end{aligned}
$$

At the tree level one may consider the RG flow under Eqs. (C2b) and (C2c) only, yielding the condition Eq. (10). Importantly, in the presence of repulsive interactions, this shows that $\Delta_{t}$ is always more relevant than $\Delta_{s}$. However, their initial values and their respective distances from strong coupling will determine the nature of the pairing in the lowenergy limit.

Assuming Hubbard-like interactions, we may approximate

$$
K_{c} \approx \sqrt{\frac{1+\frac{g}{2 \pi v}}{1+\frac{3 g}{2 \pi v}}}, \quad K_{s} \approx \sqrt{\frac{1+\frac{g}{2 \pi v}}{1-\frac{g}{2 \pi v}}},
$$

and we also define $\tilde{U}=\frac{g}{2 \pi v}$. This form is reasonable, as in an experimental setup which includes metallic gates and bulk superconductors, the interactions are fairly well screened. We note that for small $\tilde{U}$, we may expand the above coefficients and approximate the previous phase boundary in a more manageable form, $\delta_{t, 0} \geqslant\left(\delta_{s, 0}\right)^{\frac{1}{1-20}}$, where once more, the ()$_{0}$ subscript refers to the bare values.
[1] N. Read and D. Green, Phys. Rev. B 61, 10267 (2000).

[2] A. Y. Kitaev, Phys.-Uspekhi 44, 131 (2001).

[3] R. M. Lutchyn, J. D. Sau, and S. Das Sarma, Phys. Rev. Lett. 105, 077001 (2010).

[4] Y. Oreg, G. Refael, and F. von Oppen, Phys. Rev. Lett. 105, 177002 (2010).

[5] A. Kitaev, Ann. Phys. 303, 2 (2003).

[6] C. Nayak, S. H. Simon, A. Stern, M. Freedman, and S. Das Sarma, Rev. Mod. Phys. 80, 1083 (2008).

[7] M. T. Deng, C. L. Yu, G. Y. Huang, M. Larsson, P. Caroff, and H. Q. Xu, Nano Lett. 12, 6414 (2012).

[8] V. Mourik, K. Zuo, S. M. Frolov, S. R. Plissard, E. P. A. M. Bakkers, and L. P. Kouwenhoven, Science 336, 1003 (2012).

[9] A. Das, Y. Ronen, Y. Most, Y. Oreg, M. Heiblum, and H. Shtrikman, Nat. Phys. 8, 887 (2012), article .
[10] M. T. Deng, S. Vaitiekenas, E. B. Hansen, J. Danon, M. Leijnse, K. Flensberg, J. Nygård, P. Krogstrup, and C. M. Marcus, Science 354, 1557 (2016).

[11] R. Lutchyn, E. Bakkers, L. Kouwenhoven, P. Krogstrup, C. Marcus, and Y. Oreg, Nat. Rev. Mater. 3, 52 (2018).

[12] S. Iijima, Nature (London) 354, 56 (1991).

[13] J.-C. Charlier, X. Blase, and S. Roche, Rev. Mod. Phys. 79, 677 (2007).

[14] M. S. Dresselhaus, G. Dresselhaus, and R. Saito, Carbon Nanotubes, 33, 883 (1995).

[15] E. A. Laird, F. Kuemmeth, G. A. Steele, K. Grove-Rasmussen, J. Nygård, K. Flensberg, and L. P. Kouwenhoven, Rev. Mod. Phys. 87, 703 (2015).

[16] J. D. Sau and S. Tewari, Phys. Rev. B 88, 054503 (2013). 
[17] M. Marganska, L. Milz, W. Izumida, C. Strunk, and M. Grifoni, Phys. Rev. B 97, 075141 (2018).

[18] J. Klinovaja, S. Gangadharaiah, and D. Loss, Phys. Rev. Lett. 108, 196804 (2012).

[19] M. M. Desjardins, L. C. Contamin, M. R. Delbecq, M. C. Dartiailh, L. E. Bruhat, T. Cubaynes, J. J. Viennot, F. Mallet, S. Rohart, A. Thiaville, A. Cottet, and T. Kontos, Nat. Mater. 18, 1060 (2019)

[20] X.-L. Qi, T. L. Hughes, and S.-C. Zhang, Phys. Rev. B 81, 134508 (2010).

[21] A. Haim and Y. Oreg, Phys. Rep. 825, 1 (2019).

[22] G. Dresselhaus, S. Riichiro et al., Physical Properties of Carbon Nanotubes (World Scientific, Singapore, 1998).

[23] R. Peierls, Z. Phys. 80, 763 (1933).

[24] T. Ando, J. Phys. Soc. Jpn. 69, 1757 (2000).

[25] D. Huertas-Hernando, F. Guinea, and A. Brataas, Phys. Rev. B 74, 155426 (2006).

[26] F. Kuemmeth, S. Ilani, D. C. Ralph, and P. L. McEuen, Nature (London) 452, 448 (2008).

[27] W. Izumida, K. Sato, and R. Saito, J. Phys. Soc. Jpn. 78, 074707 (2009).

[28] J.-S. Jeong and H.-W. Lee, Phys. Rev. B 80, 075409 (2009).

[29] Z. Y. Zhu, Y. C. Cheng, and U. Schwingenschlögl, Phys. Rev. B 84, 153402 (2011).

[30] D. Xiao, G.-B. Liu, W. Feng, X. Xu, and W. Yao, Phys. Rev. Lett. 108, 196802 (2012).

[31] J. M. Lu, O. Zheliuk, I. Leermakers, N. F. Q. Yuan, U. Zeitler, K. T. Law, and J. T. Ye, Science 350, 1353 (2015).

[32] Y. Saito, Y. Nakamura, M. S. Bahramy, Y. Kohama, J. Ye, Y. Kasahara, Y. Nakagawa, M. Onga, M. Tokunaga, T. Nojima, Y. Yanase, and Y. Iwasa, Nat. Phys. 12, 144 (2015).

[33] X. Xi, Z. Wang, W. Zhao, J.-H. Park, K. T. Law, H. Berger, L. Forró, J. Shan, and K. F. Mak, Nat. Phys. 12, 139 (2016).

[34] T. Dvir, F. Massee, L. Attias, M. Khodas, M. Aprili, C. H. L. Quay, and H. Steinberg, Nat. Commun. 9, 598 (2018).

[35] S. C. de la Barrera, M. R. Sinko, D. P. Gopalan, N. Sivadas, K. L. Seyler, K. Watanabe, T. Taniguchi, A. W. Tsen, X. Xu, D. Xiao, and B. M. Hunt, Nat. Commun. 9, 1427 (2018).

[36] E. Sohn, X. Xi, W.-Y. He, S. Jiang, Z. Wang, K. Kang, J.-H. Park, H. Berger, L. Forró, K. T. Law, J. Shan, and K. F. Mak, Nat. Mater. 17, 504 (2018).

[37] B. T. Zhou, N. F. Q. Yuan, H.-L. Jiang, and K. T. Law, Phys. Rev. B 93, 180501(R) (2016).
[38] M. Kim, G.-H. Park, J. Lee, J. H. Lee, J. Park, H. Lee, G.-H Lee, and H.-J. Lee, Nano Lett. 17, 6125 (2017).

[39] T. Han, J. Shen, N. F. Q. Yuan, J. Lin, Z. Wu, Y. Wu, S. Xu, L. An, G. Long, Y. Wang, R. Lortz, and N. Wang, Phys. Rev. B 97, 060505(R) (2018).

[40] K. Yarimizu, H. Tomori, K. Watanabe, T. Taniguchi, and A. Kanda, J. Phys.: Conf. Ser. 969, 012147 (2018).

[41] P. Ghosh, J. D. Sau, S. Tewari, and S. Das Sarma, Phys. Rev. B 82, 184525 (2010)

[42] R. M. Lutchyn, T. D. Stanescu, and S. Das Sarma, Phys. Rev. Lett. 106, 127001 (2011).

[43] Y. Peng, F. Pientka, L. I. Glazman, and F. von Oppen, Phys. Rev. Lett. 114, 106801 (2015).

[44] D. Tománek and S. G. Louie, Phys. Rev. B 37, 8327 (1988).

[45] A. Haim, E. Berg, K. Flensberg, and Y. Oreg, Phys. Rev. B 94, 161110(R) (2016)

[46] A. Haim, A. Keselman, E. Berg, and Y. Oreg, Phys. Rev. B 89 220504(R) (2014).

[47] C. Kane, L. Balents, and M. P. A. Fisher, Phys. Rev. Lett. 79, 5086 (1997).

[48] C. L. Kane and E. J. Mele, Phys. Rev. Lett. 78, 1932 (1997).

[49] V. Fatemi, S. Wu, Y. Cao, L. Bretheau, Q. D. Gibson, K. Watanabe, T. Taniguchi, R. J. Cava, and P. Jarillo-Herrero, Science 362, 926 (2018).

[50] J. T. Ye, Y. J. Zhang, R. Akashi, M. S. Bahramy, R. Arita, and Y. Iwasa, Science 338, 1193 (2012).

[51] H. Nam, H. Chen, T. Liu, J. Kim, C. Zhang, J. Yong, T. R. Lemberger, P. A. Kratz, J. R. Kirtley, K. Moler, P. W. Adams, A. H. MacDonald, and C.-K. Shih, Proc. Natl. Acad. Sci. 113, 10513 (2016)

[52] Y. Cao, V. Fatemi, S. Fang, K. Watanabe, T. Taniguchi, E. Kaxiras, and P. Jarillo-Herrero, Nature (London) 556, 43 (2018).

[53] Y.-Z. You and A. Vishwanath, npj Quantum Mater. 4, 16 (2019).

[54] Y. Efroni, S. Ilani, and E. Berg, Phys. Rev. Lett. 119, 147704 (2017).

[55] T. Giamarchi and O. U. Press, Quantum Physics in One Dimension, International Series of Monographs (Clarendon, London, 2004).

[56] $g$ is the usual backscattering $g_{1 \perp}$ term in the standard g-ology language.

[57] J. Cardy, Scaling and Renormalization in Statistical Physics, Cambridge Lecture Notes in Physics (Cambridge University Press, Cambridge, 1996). 